\title{
Nature Neuroscience celebrates its 200th issue
}

\section{December 2014 marks the 200th issue of Nature Neuroscience. We reflect on the history of the journal and the field.}

$\mathrm{T}$ his December, Nature Neuroscience celebrates its 200th issue. Looking back at the papers published in our pages since May of 1998, when the journal was first launched, papers that are published today are increasingly more complete and multidimensional in their approaches, taking advantage of multiple cutting-edge techniques to investigate problems in depth. What used to be a cell biology study now routinely uses electrophysiological and biochemical analyses to better understand how the structure of a neuron contributes to its function. Neuroimaging studies aimed at understanding how psychological constructs are represented in the brain now include formal computational models linking them to human behavior. Looking back at the first few issues of Nature Neuroscience published in 1998, many ex vivo electrophysiology studies were self-contained units that did not report behavioral analysis in intact animals, a far cry from the papers that are published today in high-profile journals. In 1998, cognitive studies often started with sophisticated behavioral tasks, but did not often include experiments perturbing human brain function to glean insight on the brain regions responsible for the observed phenomenon.

In 2014, we see more and more neuroscience studies with multiple layers of analyses and conclusions, often in a single study. The cynics among us may bemoan this as an example of referees and editors constantly asking for more and more in the same paper, but we see this as an illustration of how much the field has matured and advanced over the last 17 years. It is not that neurons and glia have become more complex in the past two decades; they are as complicated as they have always been. But the tools wielded by neuroscientists have become more numerous and sophisticated over the years; it is now possible to ask new and interesting questions. We now have the ability to reveal brain function on a number of different levels-from insights into function to mechanisms of behavior to providing a greater understanding of the cells that comprise the basic fundamental units of the brain and the computations they perform. In addition, with the advent of molecular and genetic analyses, neuroscientists can now ask how genes and proteins at the 'omic' level contribute to neuronal and glial functions, and ultimately provide concrete links between genes, circuits and behavior. We are limited only by the questions we ask and how cleverly we design experiments to answer them.

What then can we expect to publish in our next 200 issues? As we discussed in last month's Editorial ${ }^{1}$, the focus on big data projects in neuroscience is bound to both increase the arsenal of sophisticated tools that the neuroscientist can use and create an avalanche of data that will be challenging to analyze. The traditional model of a lone scientist being able to operate independently in a small research group may not be the best approach to harness the benefits of big data ${ }^{2}$. Being able to use multiple cutting-edge techniques and being able to have a sufficiently multidisciplinary team that can tackle the integration of data across multiple levels in a single laboratory is difficult from a practical standpoint. But neuroscientists have traditionally come from different scientific training and backgrounds, and the community has always excelled at forming collaborations, so we can expect to see more labs collaborate to pool their resources and intellect. The discovery of new tools and new analytical techniques is bound to result in more fundamental discoveries about how our brain works.

As the field matures, we can also expect to see much more progress in terms of community standards for data collection and reuse. We have already begun to see more rigor in how methods are reported in papers, an initiative that Nature Neuroscience has spearheaded with our mandatory methods reporting checklist. As in other fields, such as genomics, we hope that there will be greater consensus on how different types of data should be collected and deposited, and that data descriptors (such as those available in Scientific Data) and data reuse become the norm in neuroscience. We look forward to aiding the community in these efforts.

To mark our 200th issue, we asked our readers to submit potential cover images that depicted their view of the future of neuroscience. The winning image comes from Dr. Michael Henderson of the University of Pennsylvania, depicting a mosaic comprising 320 images of cultured neurons making up a hypothetical neuron in a fractal-like fashion. We felt the image conveyed what we can expect to see in the future of neuroscience, layer after layer of analyses to provide a comprehensive understanding of the brain. We thank all of our readers who participated in the contest and congratulate the winner.

Finally, this issue marks a transition for the Nature Neuroscience editorial team. This issue is the last one finalized by its current chief editor, Kalyani Narasimhan, who was one of the three founding editors of Nature Neuroscience at its launch in May 1998. She has seen the journal from its first issue to its 200th, albeit with a brief hiatus at Nature and elsewhere. Although she is stepping down as chief editor, she will remain at Nature Publishing Group as Executive Editor, overseeing all the Nature Life Science Research journals, including Nature Neuroscience.

We are pleased to announce that the fourth chief editor of the journal, beginning with its next issue, will be Meredith LeMasurier, who started her editorial career with Neuron. We are confident that the journal will remain in good and experienced hands, and look forward to the great new neuroscience discoveries that will grace the pages of our next 200 issues.

1. Anonymous. Focus on big data. Nat. Neurosci. 17, 1429 (2014).

2. Anonymous. Consorting with big science. Nat. Neurosci. 17, 1289 (2014). 